\title{
Focus on orthopaedics
}

\section{Success in innovation but lacking consistency in established practice}

The three articles that provide the 'focus' for this issue describe the current state of orthopaedic surgery in University Hospitals of Morecambe Bay (UHMB). It is good to celebrate the success of an individual team. The authors describe the results of 59 cases of anterior cruciate reconstruction over an 18-month period, observing that early referral leads to better outcome. In a second article, the use of a locking humeral nail to treat 19 patients with humeral fractures is described. The authors claim a $100 \%$ rate in achieving a satisfactory range of shoulder movement and advocate the technique of locking only the proximal part of the nail, relying on impaction of the distal nail and a short period of postoperative immobilisation to achieve rotatory stability.

The third article reflects upon well-established and an often overlooked area of orthopaedic practice: the treatment of fragility fractures of the femur. Nationally the figures are challenging: some 60,000 patients a year in the UK suffer this problem and some $9 \%$ die in the first month. The view that early operation saves lives is accepted and yet delays in operation are frequent and cause distress to patients and relatives. The authors claim that results in Lancaster are very much 'average': an unnecessary number of patients are delayed. Many attempts to address the problem have been tried over the years. What seems to be lacking is a collective will to overcome the factors that lead to delay. In the past UHMB has employed nurse specialists to oversee a seamless service at a time when junior doctors' hours are being progressively reduced. Such a service, which was valuable while it lasted, has been discontinued. Other attempts, for example to obtain a consensus on echocardiography or provide a lead clinician with the authority to transform the service, have not come to fulfilment. There are many confounding factors: the volume of trauma and the unpredictable nature of it has to be considered, but this audit should encourage all those involved to consider that the Trust needs to take ownership of this problem. It is hoped that the new National Hip Fracture Network will galvanise jaded interest.

\section{Outcome of anterior cruciate ligament reconstruction in a district general hospital}

\section{An audit}

\author{
Mathias Nagy, MD; Sandeep Munshi, MRCS; Gaurav Rathore, MRCS; Nadeem Baqai, FRCS
}

\section{INTRODUCTION}

The primary aim of this audit was to evaluate the outcome of anterior cruciate ligament $(\mathrm{ACL})$ reconstruction in Furness General Hospital $(\mathrm{FGH})$ and to compare our results with the guideline of the British Orthopaedic Association (BOA) and current literature.

Despite the fact that $A C L$ reconstruction is a common procedure, no clear guidelines regarding the timing of reconstruction has been established. ${ }^{(I)}$ Delay in referring a patient with $A C L$ injury to a consultant orthopaedic clinic increases the risk of additional intra-articular derangements.

An ACL injury leads to instability and can cause sudden, pivoting movements in the knee. This makes the knee more prone for developing arthritis and damage to cartilage and meniscus. (2)

\section{Audit Standards}

Referral in four weeks to a consultant orthopaedic surgeon after injury

2. Government target of 18 weeks for first consultation, diagnosis and treatment

3. Physiotherapy postoperatively and at least six months follow-up using an accepted assessment system

\section{BACKGROUND}

The BOA published guidelines of best practice for primary $A C L$ reconstruction which identified best practice in general terms. Recommendations had been made regarding indication, diagnosis, pre-operative management, operative treatment and postoperative care. The prime indication for $\mathrm{ACL}$ reconstruction was symptomatic instability. Although early operative stabilisation was recommended, government targets for the first NHS outpatient appointment were difficult to achieve, due to the shortage of consultant orthopaedic surgeons in the UK. This may lead to the delay in recognition of the injury and an increase of meniscal pathology. Moreover, excessive waiting list delay for a patient with functional instability may worsen the outcome of surgery by causing additional damage to the menisci. ${ }^{(3)}$

Further recommendations included surgery on a day-case basis, the use of autografts such as 'bone-patellar-tendon-bone' or four-strand hamstring, suitable protocols designed to return the patient to their pre-injury level of functional activity (physiotherapy), and for best practice longterm follow-up should be undertaken using an accepted assessment system. ${ }^{(3)}$

The aim of $A C L$ reconstruction was to restore functional stability of the knee without compromising other joint functions. Long delay in reconstruction for a patient with 
functional instability may influence the outcome of surgery by causing additional intra-articular injuries to the menisci and cartilage. ${ }^{(4,5,6,7)}$

Different studies analysed the incidence of meniscus injuries and the timing of surgery, and recommended early rather than late $A C L$ reconstruction of the affected knee joint. ${ }^{(1,8,9,10}$ Therefore, our audit standards were: early recognition of the injury; early referral and assessment by a consultant orthopaedic surgeon; also, early ACL reconstruction and appropriate longterm follow-up, including physiotherapy and regular assessments using a standard knee scoring system.

\section{METHODOLOGY}

We carried out a retrospective review of all $\mathrm{ACL}$ reconstructions in FGH performed between July 2007 and
November 2008. We identified 59 patients, for whom 50 casenotes were available.

Our audit included 42 male patients (range: 17-42 years; mean: 27.6 years) and eight female patients (range: $15-52$ years; mean: 28.7 years). Right knee was affected in $62 \%$ $(n=31)$ of the cases.

We reviewed pre-operative clinical findings (as per the casenotes), intra-operative arthroscopic findings, magnetic resonance imaging (MRI) films and radiological reports. Outcomes were assessed using the Lysholm Knee Scoring System, which included the following outcome parameters: presence of limp, ability to weight bear, climbing stairs, squatting, instability, pain, swelling and atrophy of thigh.

Diagnosis was based on MRI scan in 37 patients, and arthroscopically in 13 patients.

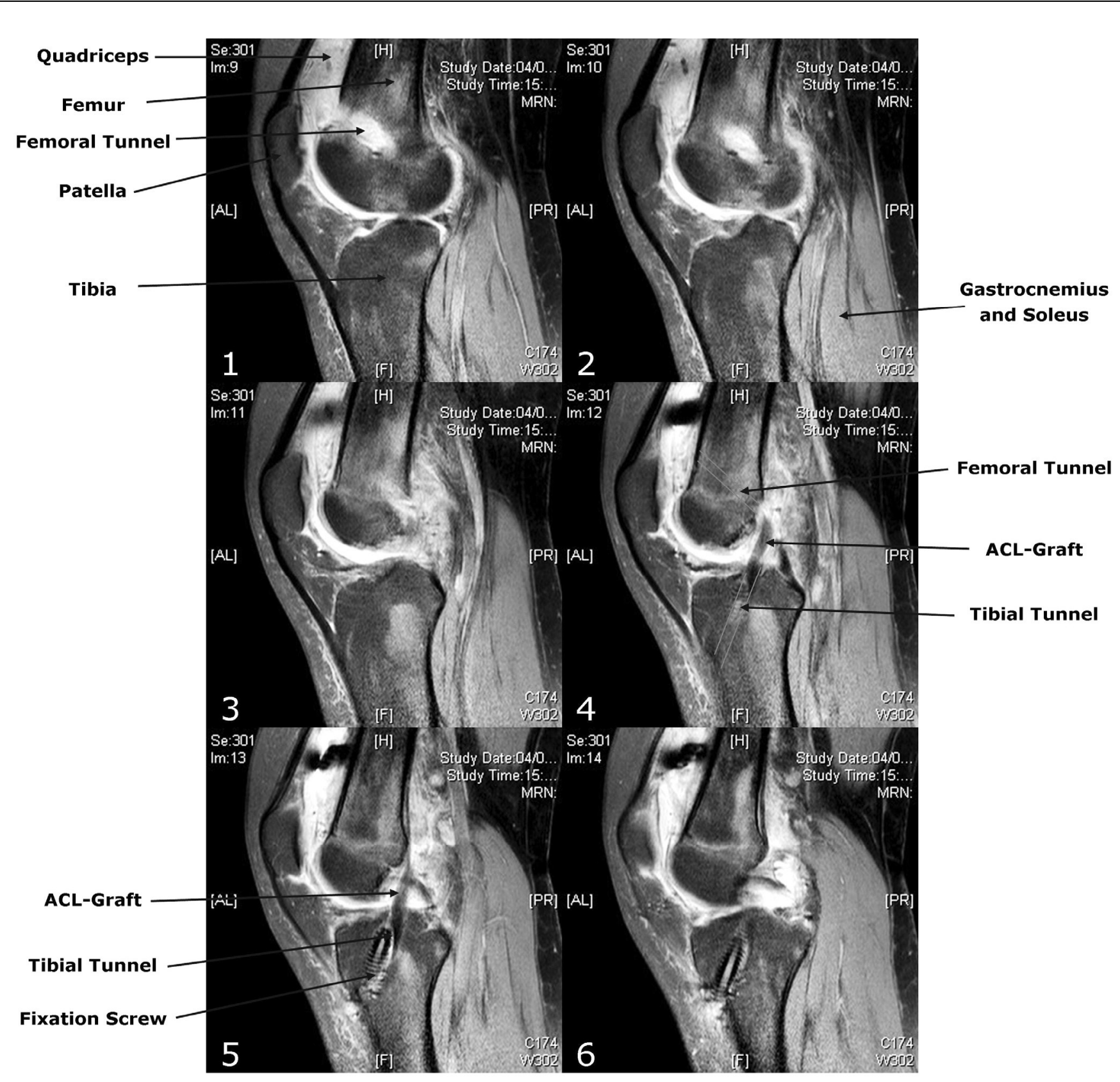

Figure I Magnetic resonance scan showing sagittal sections through the knee joint. The relationship of the ACL graft to the tunnels which have been constructed through the tibia and the femur is demonstrated 
All the operations were carried out by a single consultant surgeon using same technique: $\mathrm{ACL}$ reconstruction was performed arthroscopically taking semitendinosis, gracilis quadruple loop graft, fixing it with endo-button and absorbable Biolok ${ }^{\circledR}$ tapered screw. Following this operation all patients attended a standard rehabilitation programme.

Figure I shows MRI images of a patient following ACL reconstruction and demonstrates the placement of the femoral and tibial tunnel, graft and graft fixation.

The cause of injury in the majority of patients was due to sporting activities such as playing football (43\%), rugby (14\%) or skiing (8\%) - see figure 2 .

Orthopaedic referrals came via Accident and Emergency (A\&E) and general practitioners (GP), which accounted for $46 \%(n=23)$ and $52 \%(n=26)$ of the referrals respectively. One patient was referred from a foreign clinic.

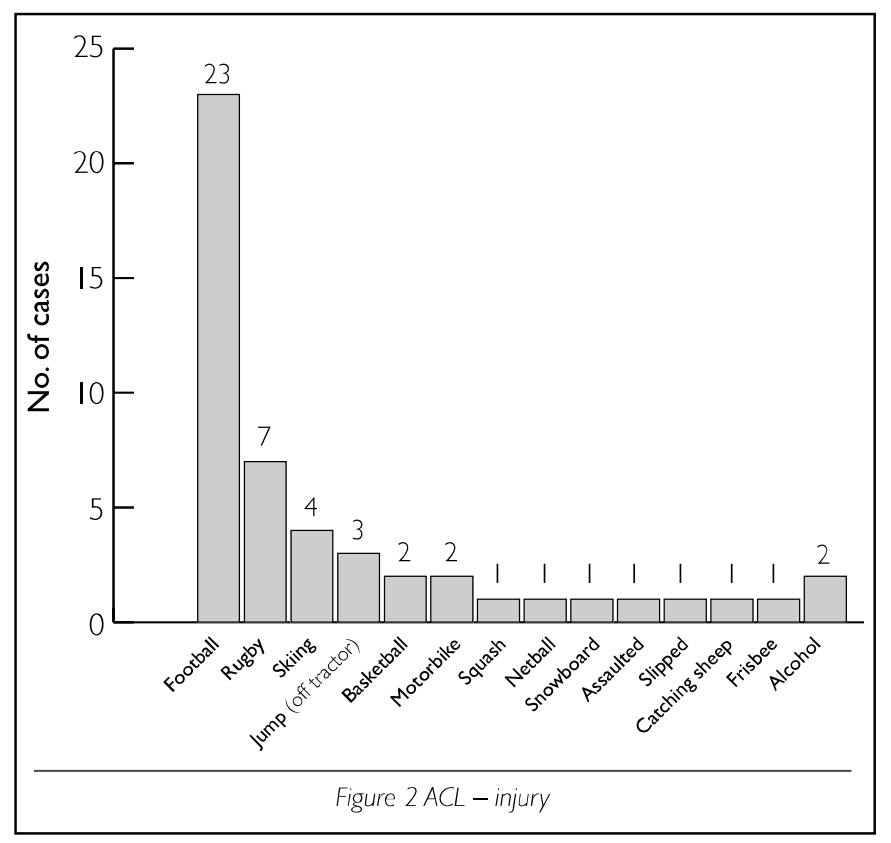

\section{RESULTS}

We determined the time span between initial injury and patient's first contact with an orthopaedic surgeon after referrals from GP or A\&E.This revealed a considerable difference. Patients referred from A\&E were seen in the orthopaedic clinic on an average in ten days (range 0-80 days) after injury. However, average duration for patients referred from GP before being seen in the orthopaedic clinic was 23 months (range I-72 months). To avoid distortion one patient was not considered. (See figure 3.)

The mean waiting time for an MRI requested by the orthopaedic team was 47 days. The average waiting time between $\mathrm{MRI}$ and operation was 176 days.

On analysing the MRI scans it was found that there was a difference regarding the presence of additional intra-articular injuries (lateral meniscus (LM) and medial meniscus (MM) injuries) in patients with early and delayed referrals. Patients referred from $A \& E$ had $44 \%$ of MM injuries and 19\% LM injuries, but in patients referred from GP the MM injury was present in $65 \%$ and LM injury in $35 \%$ of the cases (see table I).

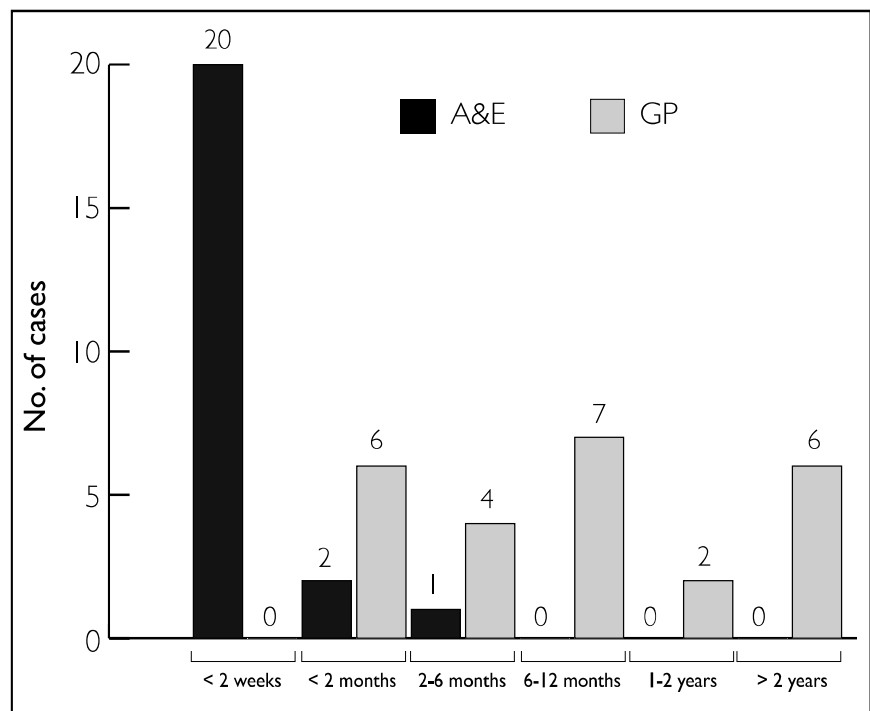

Figure 3 Injury - first seen by orthopaedic team

Findings during arthroscopic $A C L$ reconstruction, which was carried out 176 days after MRI scan on average, revealed differences in terms of presence of additional intra-articular derangements as MM and LM injuries.

Patients with early referral had less additional MM injuries (30\%) compared to patients with delayed referral, who had $46 \%$ of additional MM injuries diagnosed arthroscopically. Additional LM injuries were $31 \%$ and $43 \%$ in patients with early and late referral respectively. (See table 2.)

\begin{tabular}{|l|c|c|}
\hline & $\begin{array}{c}\text { Referred from A\&E } \\
(\mathrm{n}=23)\end{array}$ & $\begin{array}{c}\text { Referred from GP } \\
(\mathrm{n}=26)\end{array}$ \\
\hline No MRI & 7 & 6 \\
\hline MM injury & $44 \%$ & $65 \%$ \\
\hline LM injury & $19 \%$ & $35 \%$ \\
\hline \multicolumn{3}{|c|}{ Table I MRI findings } \\
\hline
\end{tabular}

\begin{tabular}{|l|c|c|}
\hline & $\begin{array}{c}\text { Referred from A\&E } \\
(n=23)\end{array}$ & $\begin{array}{c}\text { Referred from GP } \\
(n=26)\end{array}$ \\
\hline MM injury & $30 \%$ & $46 \%$ \\
\hline LM injury & $31 \%$ & $43 \%$ \\
\hline \multicolumn{2}{|c|}{ Table 2 Arthroscopic findings during ACL reconstruction } \\
\hline
\end{tabular}

Complications included superficial wound infection in two patients. A further two patients had to be re-admitted because of infection, one of whom was treated with intravenous antibiotic and the other needed wound washout. Both healed eventually.

The Lysholm Knee Scoring system was used to assess the function of the affected knee joint. We calculated this score for 12 patients in both groups (early and delayed referrals) preoperatively and following ACL reconstruction.

Our audit demonstrated that those referred from A\&E had better results pre-operatively (average 56.7) and post- 
operatively (average 95.5) and those referred from GP had average Lysholm scores of 50.4 pre-operatively and 90.7 postoperatively.

Patients referred from A\&E had more 'excellent' and 'good to excellent' results when compared with patients referred from GP where patients had more 'fair' and 'fair to good' results postoperatively. (See figure 4.)

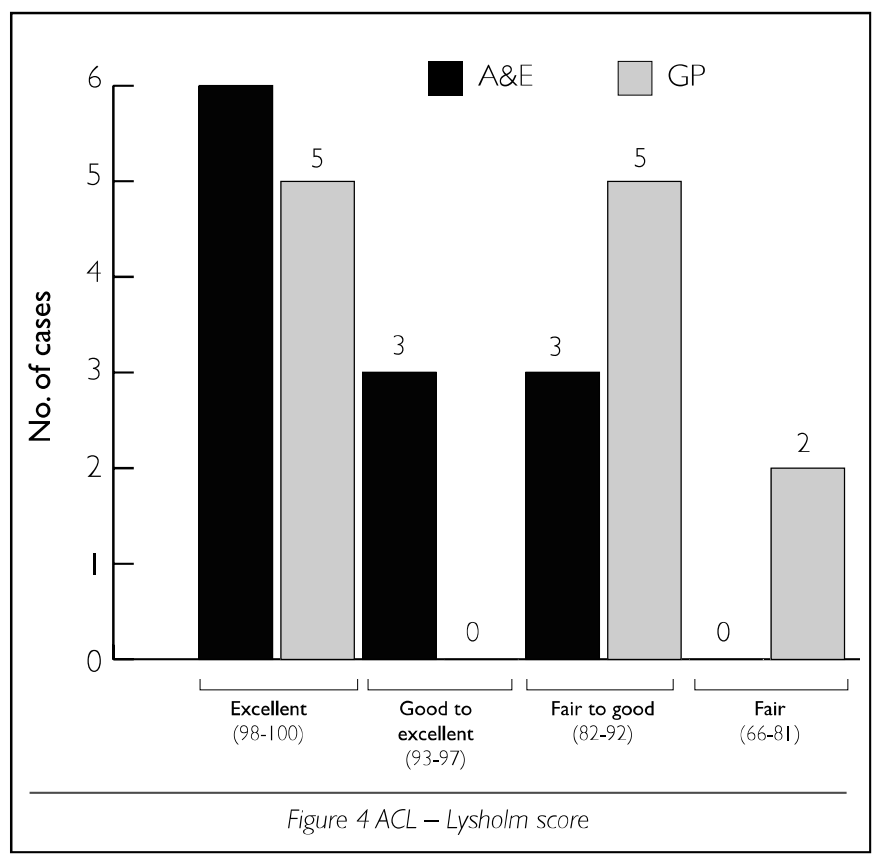

\section{DISCUSSION AND CONCLUSION}

Our audit was mainly carried out to determine the outcome of $A C L$ reconstruction and source of referral. Also, we were interested in knowing the delay that occurs between the first assessment of suspected $\mathrm{ACL}$ injury and the actual diagnosis by the orthopaedic team. We were also keen on knowing the effect of delayed diagnosis on causing further internal derangements to the knee.

Our results demonstrated that the outcome scores were better in patients with early referral (nine 'good to excellent' results) compared to only five in those with delayed referral.

We also noticed that there were higher incidences of meniscus injuries (including both MM and LM injuries) in the GP group when compared to the A\&E group, based on both MRI and arthroscopic findings.

There were clear suggestions that delay in final diagnosis and treatment was much longer in the GP group than the A\&E group.

Our early results do support the case for early referral, diagnosis and treatment of ACL injuries. We believe this leads to better outcomes and can prevent further intra-articular pathologies of the knee joint.

\section{ACKNOWLEDGEMENTS}

Many thanks to all members of the Audit Office in FGH for their outstanding support, and also the Radiology Department in $\mathrm{FGH}$.

\section{REFERENCES}

I. Papastergiou SG, Koukoulias NE, Mikalef P, Ziogas E, Voulgaropoulos $\mathrm{H}$. Meniscal tears in the ACL-deficient knee: correlation between meniscal tears and the timing of $\mathrm{ACL}$ reconstruction. Knee Surg Sports Traumatol Arthrosc 2007;15(I2):1438-44

2. Fithian DC, Paxton LW, Goltz DH. Fate of the anterior cruciate ligament-injured knee. Orthop Clin North Am 2002;33(4):62I-36

3. British Orthopaedic Association. Best practice for primary isolated anterior cruciate ligament reconstruction.

Available at:

www.boa.ac.uk/PDF\%20files/BASK/ACL\%20practice.pdf

4. Irvine GB, Glasgow MMS. The natural history of the meniscus in anterior cruciate insufficiency. J Bone Joint Surg Br 1992;74-B; 403-5

5. Shelbourne KD, Gray T. Results of anterior cruciate ligament reconstruction based on meniscus and articular cartilage status at the time of surgery: Five- to fifteen-year evaluations. Am J Sports Med 2000;28(4):446-52

6. Jomha NM, Borton DC, Clingeleffer AJ, Pinczewski LA. Long-term osteoarthritic changes in anterior cruciate ligament reconstructed knees. Clin Orthop Relat Res 1999;358: 188-93

7. Mitsou A, Vallianatos P. Meniscal injuries associated with rupture of the anterior cruciate ligament: a retrospective study. Injury 1988; 19(6):429-31

8. Church S, Keating JF. Reconstruction of the anterior cruciate ligament. Timing of surgery and the incidence of meniscal tears and degenerative change.J Bone Joint Surg Br 2005;87-B: I 639-42

9. Cipolla M, Scala A, Gianni E, Puddu G. Different patterns of meniscal tears in acute anterior cruciate ligament ruptures and in chronic ACL-deficient knees. Classification, staging and timing of treatment. Knee Surg Sports Traumatol Arthrosc 1995;3(3): I 30-4

10. Tandogan RN, Taser O, Kayaalp A, et al. Analysis of meniscal and chondral lesions accompanying anterior cruciate ligament tears: relationship with age, time from injury, and level of sport. Knee Surg Sports Traumatol Arthrosc 2004; 12(4):262-70 